\title{
Initial Results in Underwater Single Image Dehazing
}

\author{
Nicholas Carlevaris-Bianco*, Anush Mohan*, Ryan M. Eustice ${ }^{\dagger}$ \\ Department of Electrical Engineering and Computer Science* \\ Department of Naval Architecture and Marine Engineering ${ }^{\dagger}$ \\ University of Michigan, Ann Arbor, Michigan 48109 \\ Email: $\{$ carlevar, anushm, eustice\}@umich.edu
}

\begin{abstract}
As light is transmitted from subject to observer it is absorbed and scattered by the medium it passes through. In mediums with large suspended particles, such as fog or turbid water, the effect of scattering can drastically decrease the quality of images. In this paper we present an algorithm for removing the effects of light scattering, referred to as dehazing, in underwater images. Our key contribution is to propose a simple, yet effective, prior that exploits the strong difference in attenuation between the three image color channels in water to estimate the depth of the scene. We then use this estimate to reduce the spatially varying effect of haze in the image. Our method works with a single image and does not require any specialized hardware or prior knowledge of the scene. As a by-product of the dehazing process, an up-to-scale depth map of the scene is produced. We present results over multiple real underwater images and over a controlled test set where the target distance and true colors are known.
\end{abstract}

\section{INTRODUCTION}

Poor visibility due to haze can severely degrade the quality of an image. In outdoor and underwater images, the light reflected from the subject and ambient light in the medium, referred to as the airlight, are absorbed and scattered by particles in the medium before they reach the camera. Depending on the density and size of the suspended particles, this can result in severely degraded images. Dehazing serves to improve the aesthetic quality of images as well as to improve data quality for scientific data collection and computer vision applications.

Our aim in this paper is to explore single image dehazing techniques for underwater images (Fig. 1). While a number of single image dehazing methods have shown good performance in outdoor images, much remains to be said about the success of these techniques for underwater images. We present a method for single image dehazing specifically designed for underwater images. Our main contribution is the proposal of a depth prior that exploits the wavelength-dependent attenuation of light in water to estimate the depth of a scene from a single image.

\section{A. Optical Model}

The image captured by a camera can be modeled as being composed of two components: the direct transmission of light from the object and the transmission due to scattering by the particles of the medium, referred to here as the airlight. Mathematically, this can be written as [1,2]:

$$
I(\mathbf{x})=J(\mathbf{x}) t(\mathbf{x})+(1-t(\mathbf{x})) A
$$

where $\mathbf{x}=(x, y)$ is a pixel, $I(\mathbf{x})$ is the observed image intensity, $J(\mathbf{x})$ is the scene radiance, $A$ is the airlight and $t(\mathbf{x})$ is the transmission. The transmission is based on the Lambert-Beer law for transparent objects, which states that light traveling through a transparent material will be attenuated exponentially [1,2]:

$$
t(\mathbf{x})=\exp (-\beta d(\mathbf{x})) .
$$

Here, $d(\mathbf{x})$ is the scene depth and $\beta$ is the attenuation coefficient due to scattering in the medium. Note that the observed image, $I(\mathbf{x})$, scene radiance, $J(\mathbf{x})$, and airlight, $A$, are all vectors in $\Re^{3}$ with one intensity value per color channel. The attenuation coefficient due to scattering, $\beta$, is not a function of the color channel and therefore, for a given pixel, the transmission is constant over all three color channels.

Assuming that we can obtain a good estimate of the airlight, $A$, there are two unknowns in (1) that we need to solve for: the transmission, $t(\mathbf{x})$, which captures the depth of the scene, and $J(\mathbf{x})$, the clear image. This leads to an inherent ambiguity between deducing the scene depth from the direct transmission, which needs to be resolved. Without additional information or assumptions about the scene, one cannot determine if the color of the patch is caused by haze when the object is far away or by the natural color of the object without haze (Fig. 2).

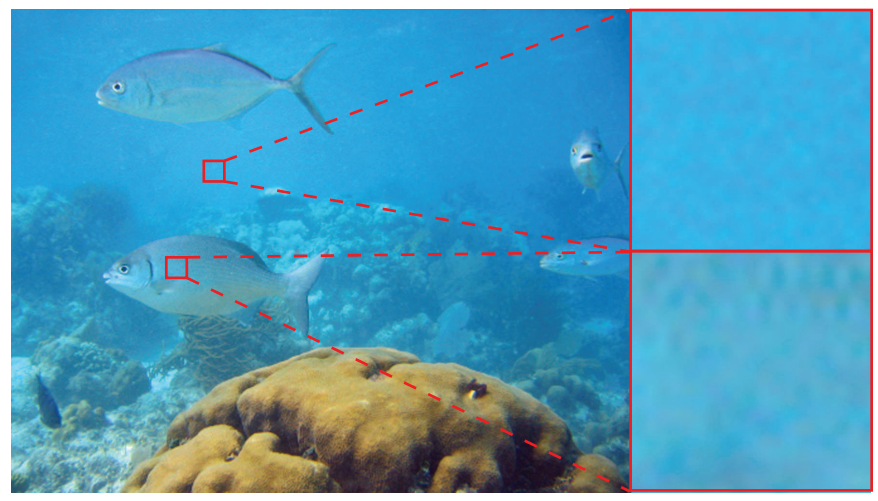

Fig. 2. There is an inherent ambiguity between the scene depth and direct transmission. Given a patch in the image, one cannot determine if the color of the patch is caused by haze when the object is far away (upper box) or by the natural color of the object without haze (lower box). 


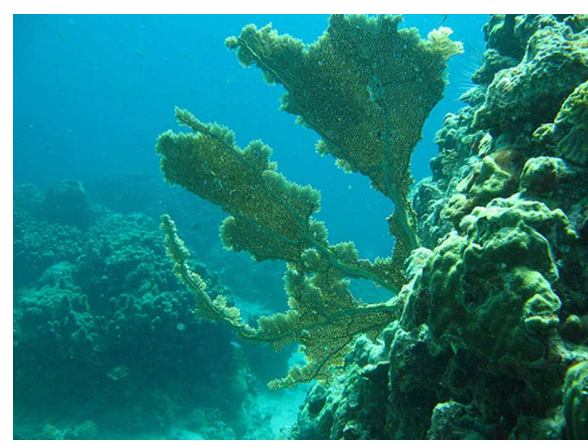

(a) Original image.

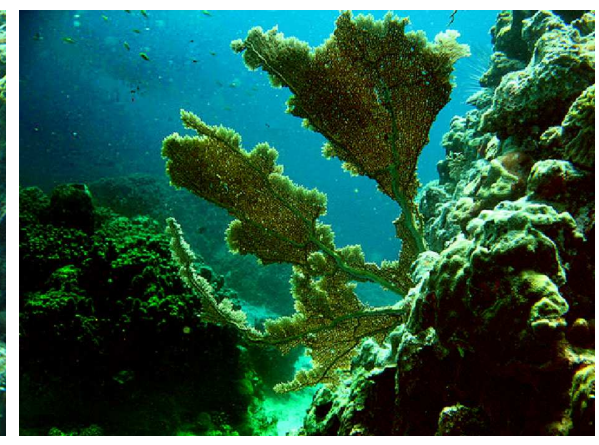

(b) Dehazed image.

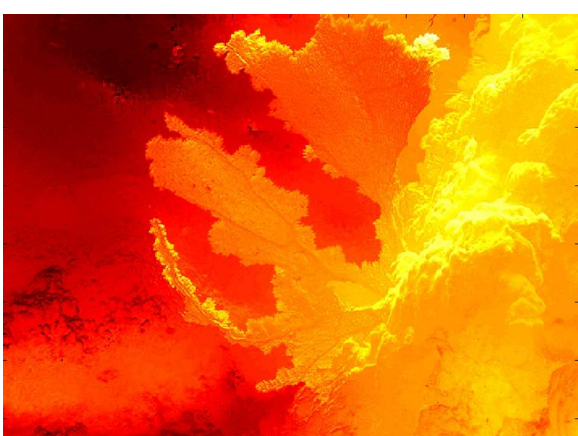

(c) Depth map.

Fig. 1. Single image dehazing for underwater images. Note the detail revealed in the upper left quarter of the dehazed image, (b).

\section{B. Previous Work}

In order to resolve the ambiguity between the depth and the direct transmission, many existing dehazing techniques rely on additional hardware such as polarization filters [3, 4], additional information such as the depth of the scene [5], or multiple images captured under different conditions [2, 6]. Very recently, several techniques have been developed to remove haze from a single outdoor image with no additional information [7-10]. These methods use statistical priors on the properties of natural images in order to remove the haze. Tan [7] proposes to recover the clear image by maximizing the contrast of the image over local patches. Fattal [8] exploits the fact that the transmission and scene albedo are locally uncorrelated to dehaze the image. He et al [9] introduce a novel dark channel prior that is a statistical prior on the minimum intensity color channel in an image patch. They exploit the fact that objects in a clear image patch have at least one color channel with very low intensity, but in a hazy patch all color channels will have higher intensity due to airlight addition. All three methods recover an up-to-scale scene depth map when they solve for the dehazed image. Kratz and Nishino [10] model the scene albedo and depth as two statistically independent layers and use a factorial Markov random field (MRF) to solve for the dehazed image. They provide a more general framework that can include different priors on depth and natural image statistics. While these single image methods have shown very good performance in terrestrial images, only Fattal provides results for underwater imagery.

\section{Comparison with comprehensive underwater model}

The model in (1) is commonly used in terrestrial image dehazing [2, 7-9]. However, a more complete underwater model is described by Duntley in $[1,11]$ :

$$
\begin{aligned}
{ }_{t} N_{r}(z, \theta, \phi)= & { }_{t} N_{0}\left(z_{t}, \theta, \phi\right) e^{(-\alpha(z) r)} \\
& +N\left(z_{t}, \theta, \phi\right) e^{(K(z, \theta, \phi) r \cos \theta)} \\
& \left(1-e^{(-\alpha(z) r+K(z, \theta, \phi) r \cos \theta)}\right) .
\end{aligned}
$$

In this section we compare the two models and explain under which assumptions the models are equivalent. In Duntley's model ${ }_{t} N_{r}(z, \theta, \phi)$ is the radiance of the target seen by the observer; it is a function of the depth of the observer, $z$, and the zenith and azimuth angles between the observer and the target, $\theta$ and $\phi$, respectively. The observed radiance is a mixture of the radiance at the subject, ${ }_{t} N_{0}\left(z_{t}, \theta, \phi\right)$, and the radiance in the water column (the airlight), $N\left(z_{t}, \theta, \phi\right)$. In these terms $z_{t}$ represents the depth of the target. The attenuation coefficient, $\alpha(z)$, is the sum of two terms, the scattering coefficient and the absorption coefficient. The distance between the observer and the target is denoted by $r$. The radiance attenuation function, $K(z, \theta, \phi)$, captures how the airlight changes with depth.

Duntley notes two possible simplifications to this model [11]. First, the attenuation coefficient, $\alpha$, is a constant if the water is uniform between the subject and observer. Second, "[b]ecause underwater sighting ranges rarely exceed $2 / K$, the effect of $K$ variation is seldom appreciable, except near the surface." Additionally, we note that when images are collected horizontally with zenith angle $\theta \approx \pi / 2$, the effect of the $K$ variation is zero. Therefore, there is a large subset of underwater images that can be modeled using a greatly simplified model under the assumptions that they have been collected in uniform water, either nearly horizontally or at sufficient depth such that vertical variation in negligible:

$$
\begin{aligned}
{ }_{t} N_{r}(z, \theta, \phi)= & { }_{t} N_{0}\left(z_{t}, \theta, \phi\right) e^{(-\alpha r)} \\
& +N\left(z_{t}, \theta, \phi\right)\left(1-e^{(-\alpha r)}\right) .
\end{aligned}
$$

Notation aside, this simplified version of the full underwater model is almost exactly equivalent to the standard model used for terrestrial dehazing, (1), with one exception. In the full underwater model the attenuation coefficient, $\alpha$, is the sum of the scattering and absorption coefficients, $\alpha=\alpha_{s}+\alpha_{a}$, while in the terrestrial model only scattering is considered. Scattering and absorption are very different physical effects. Scattering is caused by larger suspended particles in the medium and is largely wavelength independent, and therefore only a function of scene depth. Absorption, however, is a function of both scene depth and wavelength, attenuating the red color channel much more severely than blue or green. As these are two different phenomena, in this paper we only seek to correct the effects of scattering and therefore will use the dehazing model as shown in (1). 
While simplified, the assumptions in this model do not imply that our proposed method will not provide color correction for the reduction in the red channel caused by absorption. Our estimate of the airlight includes the effects of absorption on the airlight, i.e. that the airlight is blue, not white. Therefore, when we remove the additive airlight the color balance of the imagery is improved, without having to additionally account for absorption effects.

The remainder of this paper is outlined as follows. In Section II we show that one can exploit the strong difference in attenuation between image color channels to estimate the depth of the scene, an important step in dehazing the image. In Section III we discuss how the scene radiance is modeled as a Markov random field (MRF) and how a maximum a posteriori (MAP) estimate of the dehazed image is calculated using a Min Cuts / Max Flow minimization algorithm. In Section IV we propose a method for estimating the airlight and discuss our results in Section V. Finally, in Section VI we discuss future extensions of single image underwater dehazing.

\section{ESTIMATING THE DEPTH OF AN UNDERWATER SCENE}

The effects of haze are highly correlated with the range depth of the underwater scene. The further away an object is, the more hazy it will appear in the image. Therefore, an accurate estimate of the scene depth can be used to dehaze an image, assuming for now that the airlight is known. We propose a simple, yet effective, prior to estimate the scene depth of underwater images. This prior exploits the fact that the attention of light in water varies greatly depending on the color of the light. Specifically, the red color channel is attenuated at a much higher rate than green or blue.

Our prior compares the maximum intensity of the red color channel, to the maximum intensity in the green and blue color channels, over a small image patch. First, we find the difference between the maximum red channel intensity and the maximum of the green and blue channels

$$
D(\mathbf{x})=\max _{\mathbf{x} \in \Omega, c \in r} I_{c}(\mathbf{x})-\max _{\mathbf{x} \in \Omega, c \in\{b, g\}} I_{c}(\mathbf{x}) .
$$

Here $I_{c}(\mathbf{x})$ refers to a pixel $\mathbf{x}$ in color channel $c \in\{r, g, b\}$ in the observed image, and $\Omega$ refers to a patch in the image. Note that the image intensities have been normalized to values between zero and one.

The estimated transmission, $\tilde{t}$, is found by shifting the values of $D$ so that the largest difference between color channels, which represents the closest point in the foreground, is one:

$$
\tilde{t}(\mathbf{x})=D(\mathbf{x})+\left(1-\max _{\mathbf{x}} D(\mathbf{x})\right) .
$$

Because our prior is calculated over an image patch, it produces a coarse initial estimate of the depth. He et al note that a closed form solution for natural image matting [12], can be used to refine a coarse depth prior. Natural image matting seeks to solve a different yet mathematically equivalent problem, in which an image, $I$, is modeled as a linear combination of the background, $B$, and foreground, $F$. Natural image matting then seeks to determine, for each pixel, the percentage that it is composed of either the foreground or background based on sparse user input marking sample foreground and background regions. The mixture between foreground and background is captured in the foreground opacity, $\gamma$.

$$
I_{c}=\gamma_{c} F_{c}+\left(1-\gamma_{c}\right) B_{c}
$$

One can see that if we consider the foreground to be the scene radiance, and the background to be the airlight, then the foreground opacity, $\gamma$, is exactly the transmission, $t$. We, therefore, can provide our initial coarse estimate of the transmission, in place of user input, to the natural image matting algorithm to produce a refined transmission estimate. Fig. 3 shows the depth map generation process where (b) shows the initial estimate of the transmission produced by our prior and (c) shows the transmission estimate after being refined using the natural image matting method proposed by Levin et al [12].

Finally, in order to maintain realistic images, which tend to contain a small amount of haze, and to prevent the algorithm from accentuating noise in very hazy regions of the image, we place a lower bound on the estimated transmission. This lower bound is enforced through the parameter $\omega$ :

$$
\tilde{t}=\left\{\begin{array}{ll}
\tilde{t} & \text { for } \tilde{t} \geq \omega \\
\omega & \text { for } \tilde{t}<\omega
\end{array} .\right.
$$

The figures in this paper were generated with $0.75 \leq \omega \leq 0.95$ and with a square patch size $20 \leq \Omega \leq 60$ pixels depending on the size of the image. We found that larger input images required larger patch sizes.

\section{Scene Radiance Estimation}

Once we have a good estimate of the scene depth we then estimate the scene radiance. We can directly calculate $J$ from the observed image as follows:

$$
J(\mathbf{x})=\frac{I(\mathbf{x})-A}{t(\mathbf{x})}+A .
$$

However, in order to regularize our calculation of the scene radiance we model the true scene radiance, $J_{o}$, as a Markov random field observed under white Gaussian noise (WGN)

$$
J(\mathbf{x})=J_{o}(\mathbf{x})+w(\mathbf{x})
$$

where $w \sim \mathcal{N}(0,1)$. We then seek to compute the maximum a posteriori estimate of the clear image, $\tilde{J}$. This probabilistic formulation is very common in a wide variety of early vision problems [13].

In order to compute the maximum a posteriori estimate of the clear image, $\tilde{J}$, we maximize the posterior probability

$$
P\left(J_{o}(\mathbf{x}) \mid J(\mathbf{x})\right) \propto P\left(J(\mathbf{x}) \mid J_{o}(\mathbf{x})\right) P\left(J_{o}(\mathbf{x})\right) .
$$

All MRF with a first-order grid neighborhood system have a probability distribution that can be expressed as a Gibbs distribution in the form [13]

$$
P\left(J_{o}(\mathbf{x})\right)=\frac{1}{Z} \exp \left(\frac{1}{T_{0}} \sum_{\forall \mathbf{x}_{i}, \mathbf{x}_{j} \in N\left(\mathbf{x}_{i}\right)} V\left(\mathbf{x}_{i}, \mathbf{x}_{j}\right)\right)
$$




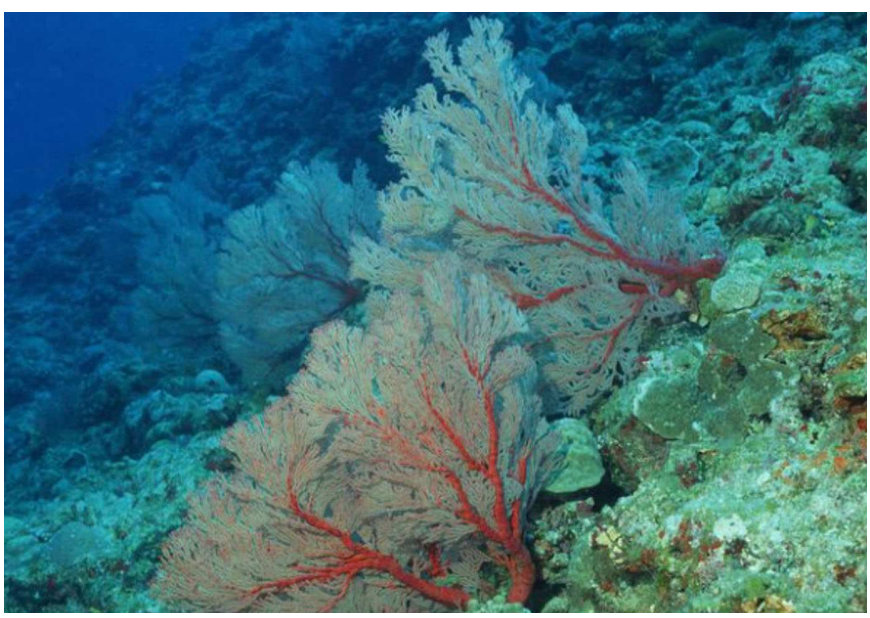

(a) Original image.

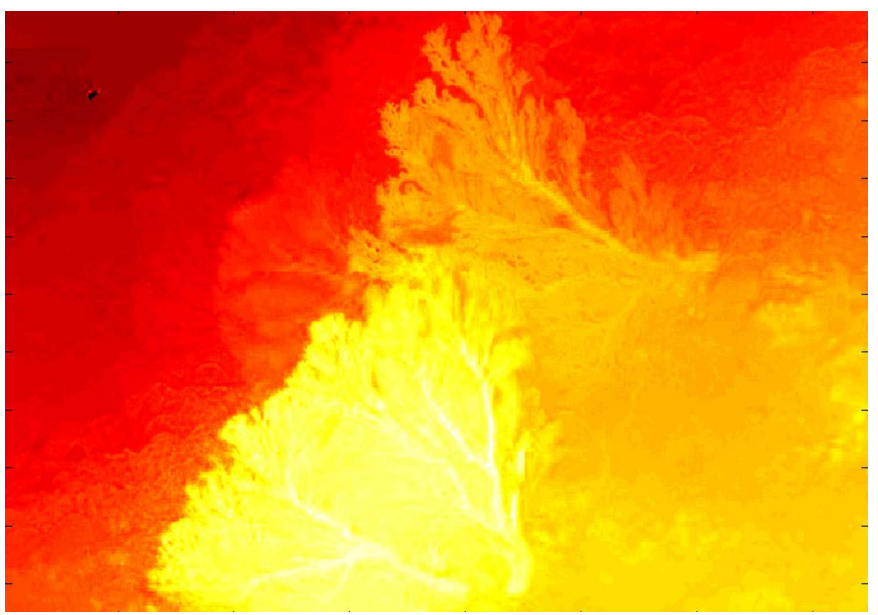

(c) Refined transmission.

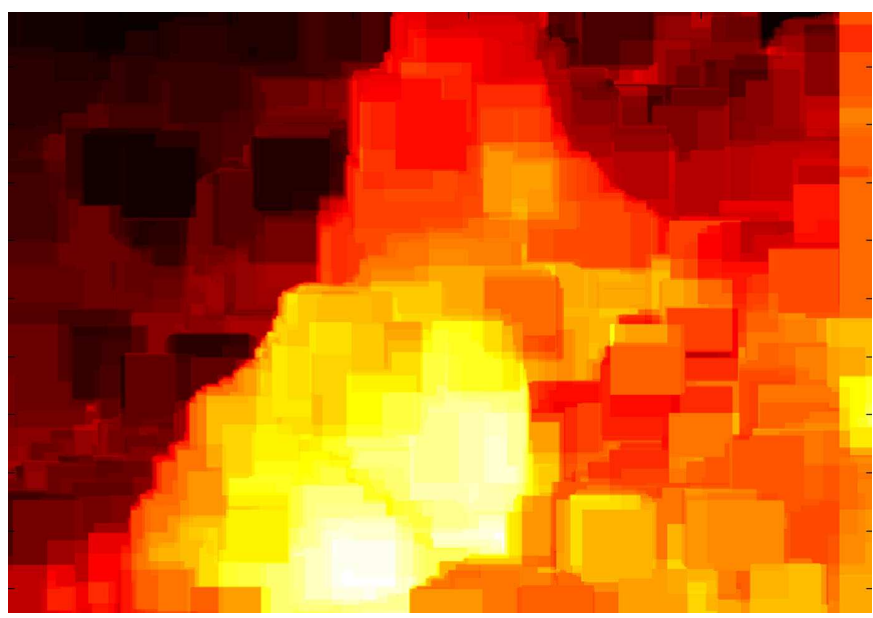

(b) Initial transmission.

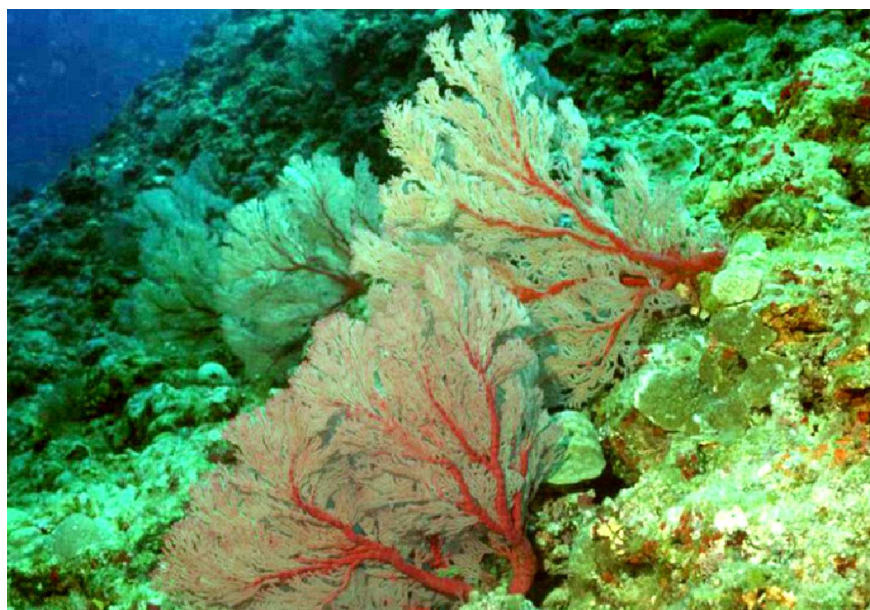

(d) Dehazed image.

Fig. 3. From the original image, (a), a coarse estimate of the scene transmission is estimated based on the difference between the maximum red and blue or green channel intensities, (b). The coarse estimate is then refined using natural image matting, (c). Finally, the clear image is estimated (d).

where $Z$ is a normalizing factor, $T_{0}$ is a free parameter referred to as the "natural temperature" or "inverse temperature," and $V$ is a potential function between a given pixel location $\mathbf{x}_{i}$ and its neighbor $\mathbf{x}_{j}$. For a potential function we selected a simple Potts model [14] to promote spatial regularity

$$
V\left(\mathbf{x}_{i}, \mathbf{x}_{j}\right)=\left\{\begin{array}{ll}
0 & \text { for } J\left(\mathbf{x}_{i}\right)=J\left(\mathbf{x}_{j}\right) \\
1 & \text { for } J\left(\mathbf{x}_{i}\right) \neq J\left(\mathbf{x}_{j}\right)
\end{array} .\right.
$$

Based on the additive noise model in (10) the likelihood of the observed scene radiance, $J$, is defined as

$$
P\left(J(\mathbf{x}) \mid J_{o}(\mathbf{x})\right)=C \exp \left(-1 / 2 \sum_{\forall \mathbf{x}_{i}}\left(J\left(\mathbf{x}_{i}\right)-J_{0}\left(\mathbf{x}_{i}\right)\right)^{2}\right)
$$

where $C$ is a normalizing constant. Therefore, we can now find the MAP estimate by minimizing the negative $\log$ of the posterior probability

$$
\begin{gathered}
\tilde{J}(\mathbf{x})=\arg \min _{J(\mathbf{x})}-\ln P\left(J(\mathbf{x}) \mid J_{\text {true }}(\mathbf{x})\right) \\
=\arg \min _{J(\mathbf{x})} \sum_{\forall \mathbf{x}_{i}}\left(J\left(\mathbf{x}_{i}\right)-J_{0}\left(\mathbf{x}_{i}\right)\right)^{2} \\
+\eta \sum_{\forall \mathbf{x}_{i}, \mathbf{x}_{j} \in N\left(\mathbf{x}_{i}\right)} V\left(\mathbf{x}_{i}, \mathbf{x}_{j}\right),
\end{gathered}
$$

where $\eta=2 / T_{0}$ is a scaling constant. One can tune $\eta$ to increase or decrease the amount of smoothness enforced during the estimation process. The figures in this paper were generated with $\eta=1$.

The minimization required in (15) can be efficiently approximated using an existing graph cut minimization algorithm [14-16].

\section{Airlight Estimation}

In the previous sections we assumed that an estimate of the airlight was known. We will now show that the airlight can be estimated using the transmission estimate, $\tilde{t}$. After the 


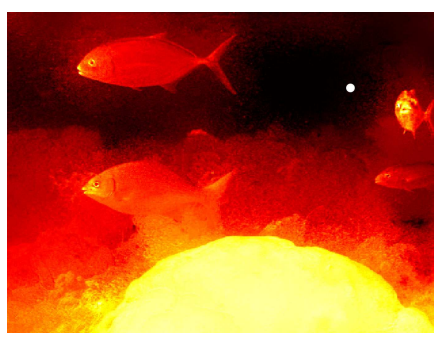

(a) Depth map.

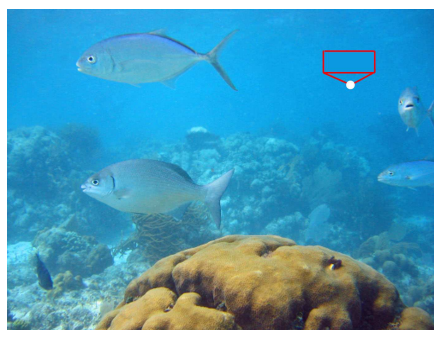

(b) Original image.

Fig. 4. The airlight is estimated by finding the minimum transmission value, marked with a white dot, in the depth map, (a). The color in the original image is sampled at this location and is used as the airlight estimate. The location in the original image, (b), is marked with a white dot and the sampled airlight color is shown in the red box.

transmission estimate has been refined, but before we enforce a lower limit on the transmission as shown in (8), we find the pixel with the minimum estimated transmission. This pixel represents the point in the image furthest from the camera. We then use the intensity values at this location in the original image as the airlight estimate.

$$
\tilde{\mathbf{A}}=I\left(\arg \min _{\mathbf{x}} \tilde{t}(\mathbf{x})\right)
$$

Fig. 4 shows an example of the location used as the airlight estimate marked as a white dot. The color sampled from the original image is shown in the red box above the sample point.

One important requirement for airlight estimation is that within the image there be a region that is completely hazeopaque. This usually occurs in a region above the horizon, in which only the water column is visible. Fig. 5 contains an image where our method may not have properly identified the airlight. In this image the pixel used for airlight estimation, marked with a white dot, is not completely haze obscured.

\section{REsults}

First we compare our result with those provided by Fattal [8] and against simple histogram equalization. Fig. 6 contains the comparison with Fattal's work for two underwater images. For the image of the fish our result provides better dehazing of the background than Fattal's result. Our result shows more red coloration in the two foremost fish than Fattal's, and without an available ground truth for the color of the fish we are not sure which is more accurate. The image of the ship provides a more difficult test. Our result exposes details in the lower left corner of the image, and along the side of the shipwreck that are less visible in Fattal's. However, our result produces an overly-bright background not present in the original nor Fattal's.

In Fig. 7 we compare our results with established histogram equalizations methods used to increase the contrast of images. For comparison we use two standard MATLAB ${ }^{\circledR}$ functions, histeq(), which performs a spatially-invariant histogram equalization, and adapthisteq(), which performs a spatially-variant adaptive histogram equalization. In both cases the image was first converted to the $L * a * b$ color

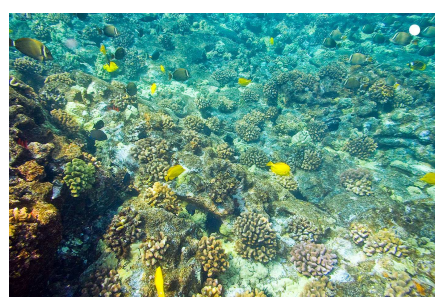

(a) Original image.

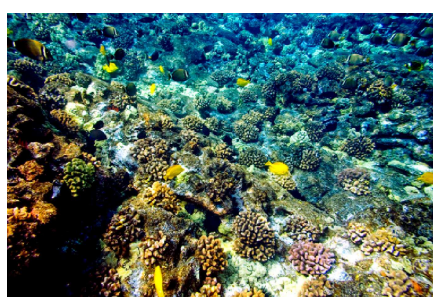

(b) Dehazed image.

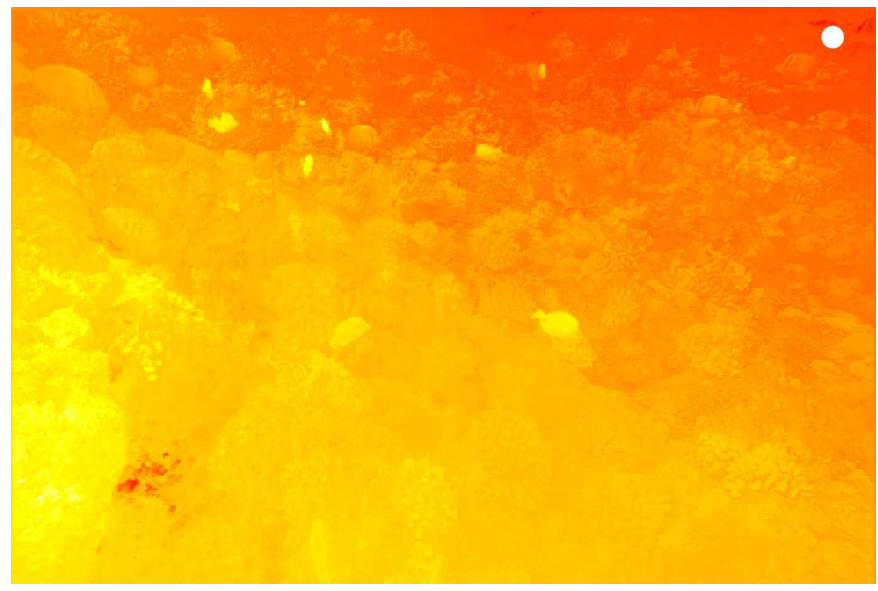

(c) Depth map.

Fig. 5. In this image the pixel use for airlight estimation, marked with a white dot in (a) and (c), is not completely haze obscured. This results in a poor airlight estimate and in turn a less-than-ideal dehazing result, (b). For best results our airlight estimation method requires a completely haze-opaque region to be visible in the original image

space and the equalizations were performed on the luminance color channel. One can see that our results reveal greater image detail than either histogram equalization results.

In order to test the color accuracy of our estimation method we collected multiple images of a color wheel target taken at varying distances between 3 and 6 meters from the camera in a fresh water test tank. We then used an image of the color wheel taken in air as the ground truth for haze correction. Fig. 8 shows the results of this experiment where 8(a) shows the original images collected at varying distances underwater, scaled to the same size. For reference, the color wheel image taken in air is shown at the far left. From each color wheel three segments are sampled and then displayed below the images to allow easier comparison between colors. Fig. 8(b) shows the images after dehazing, again with the truth color wheel on the far left, this time white balanced for better color comparison. One can see that the dehazed images have colors much closer to the truth image and that the colors are fairly consistent over the different depths. Note that in this experiment the airlight was selected manually as the images do not contain a completely haze-obscured region for the airlight estimate as discussed in Section IV.

Fig. 8(c) shows the depth map for the color wheel experiment. Our prior provides a good depth estimate of the planar target showing especially good results over the color wheel itself. One problem with the depth estimate is that the white 


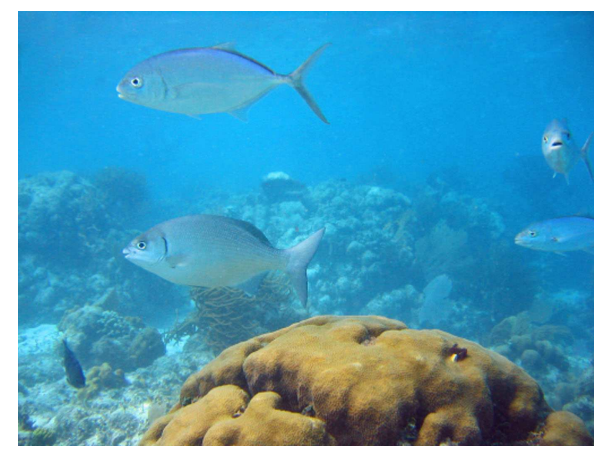

(a) Original image.

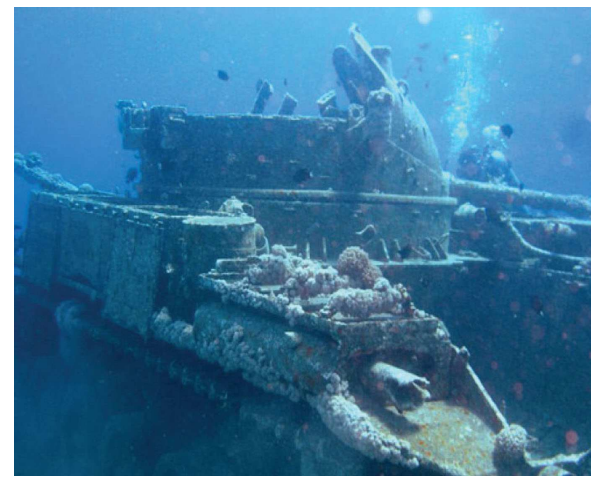

(d) Original image.

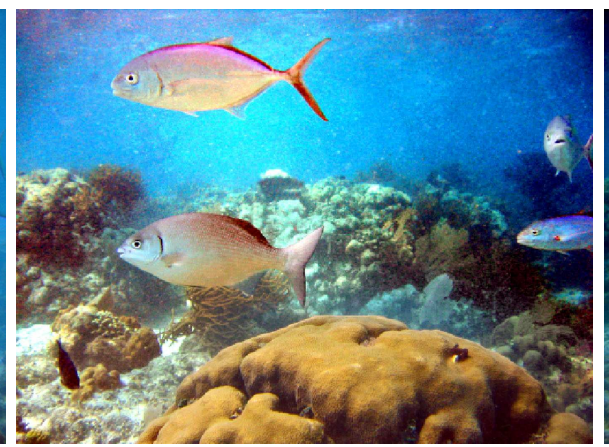

(b) Our result.

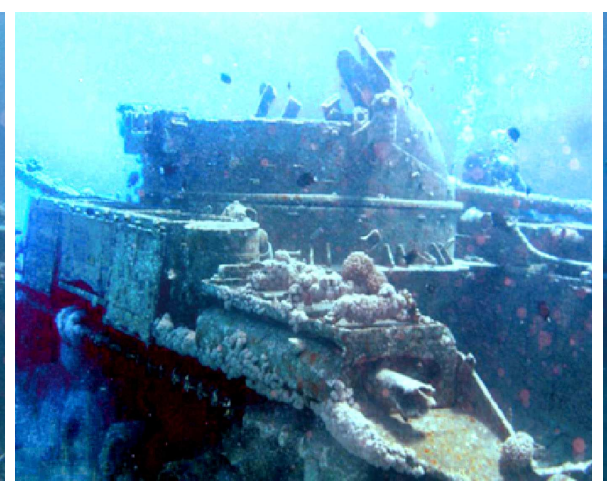

(e) Our result.

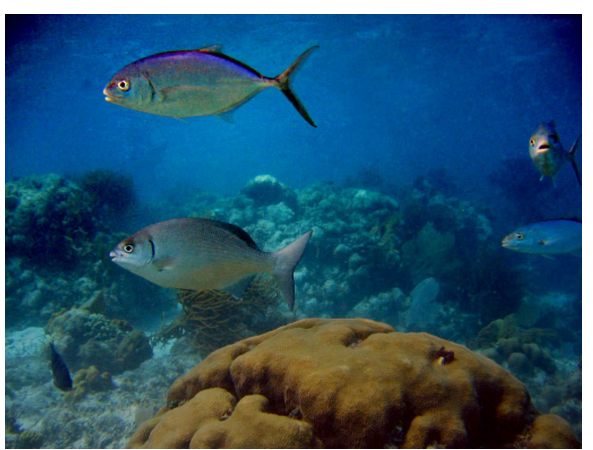

(c) Fattal's result.

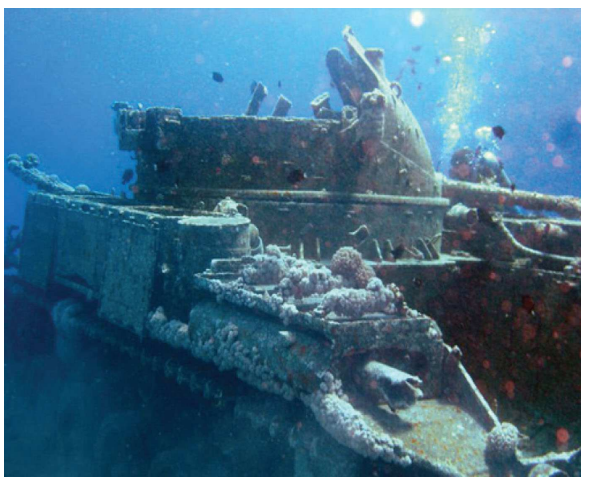

(f) Fattal's result.

Fig. 6. Comparison with Fattal's method.

region of the target surrounding the color wheel is consistently estimated to be closer than the color wheel itself. This illustrates one shortcoming of the proposed depth prior. The depth prior often has difficulty determining the depth of large solid colored objects. In most unstructured underwater environments this should not pose much of a problem. However, it may be a problem for specific applications.

\section{Vi. CONClusions AND Future Work}

In this paper we proposed an algorithm to dehaze underwater images from a single image. We presented a simple prior based on the difference in attenuation among the different color channels, which allows us to estimate the depth of a scene. We then use this depth information to recover the scene radiance from the hazy image by modeling the true scene radiance as a Markov Random Field, which can be estimated using a MAP estimator. We presented results over multiple real underwater images and over a controlled test set where the target distance and true colors are known.

Currently, our technique can reduce the effect of haze caused by the scattering of light and is capable of providing a color correction through the airlight estimate. However, the difference in absorption among the color channels does play a role in degrading underwater images. In a future work we hope to develop additional methods to compensate for attenuation in addition to scattering. We also plan to include the affects on airlight caused by changes in the water depth from the surface as described in Section I-C. This will further increase the different types of underwater images our algorithm can dehaze.

Most importantly, we note that many images collected underwater are not collected in ambient light. Often, due to the high absorption of light in water, images must be collected with artificial lighting. We hope to adapt this method to work with scenes that have been captured with artificial lighting.

\section{ACKNOWLEDGMENTS}

This work was supported by the National Science Foundation under NSF Award IIS-0746455.

\section{REFERENCES}

[1] S. Q. Duntley, A. R. Boileau, and R. W. Preisendorfer, "Image transmission by the troposphere i," J. Opt. Soc. Am., vol. 47, no. 6, pp. 499-506, 1957.

[2] S. Narasimhan and S. Nayar, "Vision and the Atmosphere," in International Journal on Computer Vision, vol. 48, no. 3, Jul 2002, pp. 233-254.

[3] Y. Y. Schechner, S. G. Narasimhan, and S. K. Nayar, "Instant dehazing of images using polarization," Computer Vision and Pattern Recognition, IEEE Computer Society Conference on, vol. 1, p. 325, 2001.

[4] S. Shwartz, E. Namer, and Y. Y. Schechner, "Blind haze separation," Computer Vision and Pattern Recognition, IEEE Computer Society Conference on, vol. 2, pp. 19841991, 2006. 


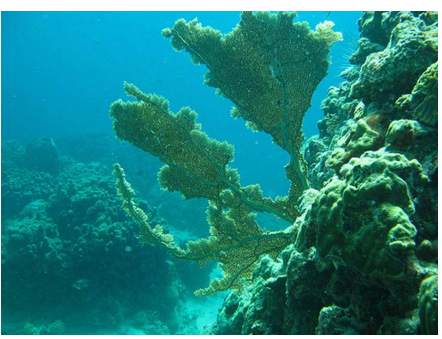

(a) Original image.

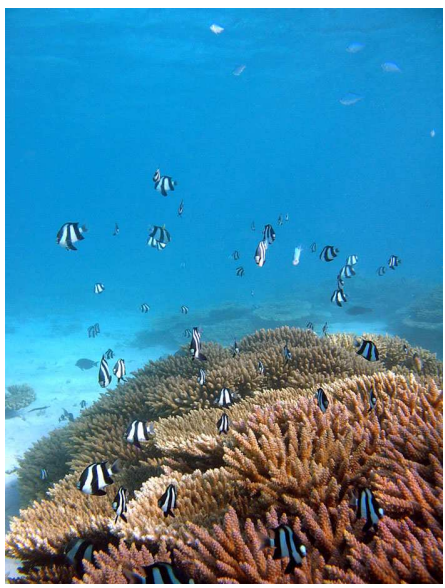

(e) Original image

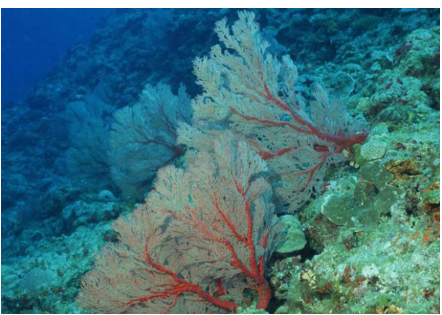

(i) Original image

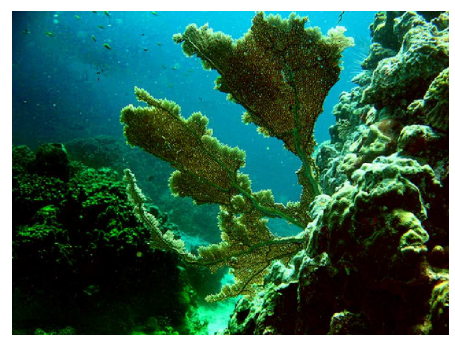

(b) Our result.

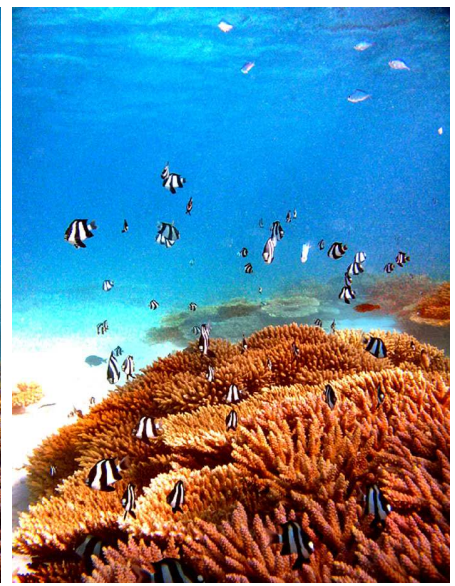

(f) Our result.

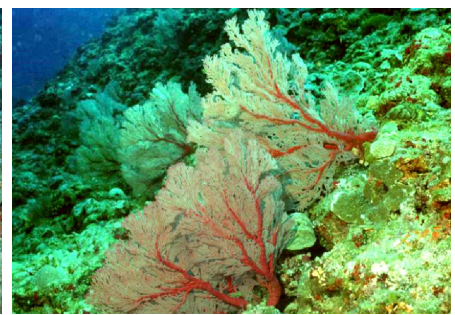

(j) Our result.

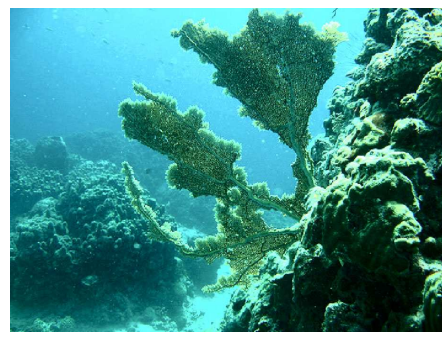

(c) histeq()

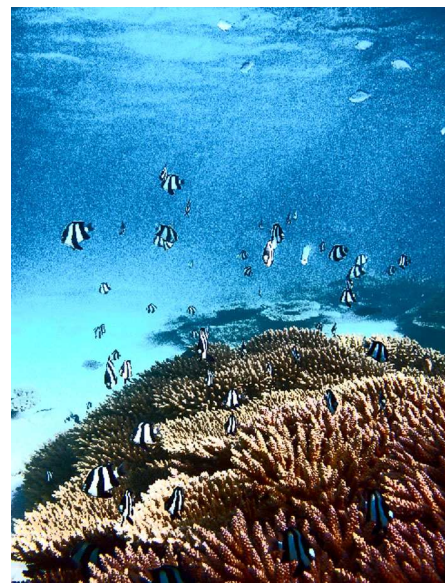

(g) histeq().

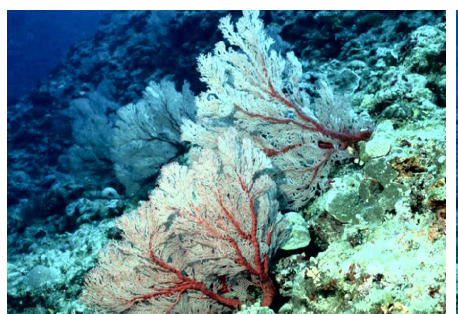

(k) histeq().

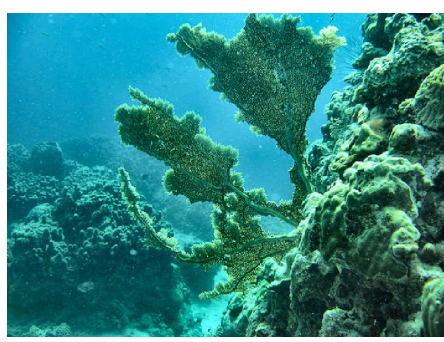

(d) adapthisteq()

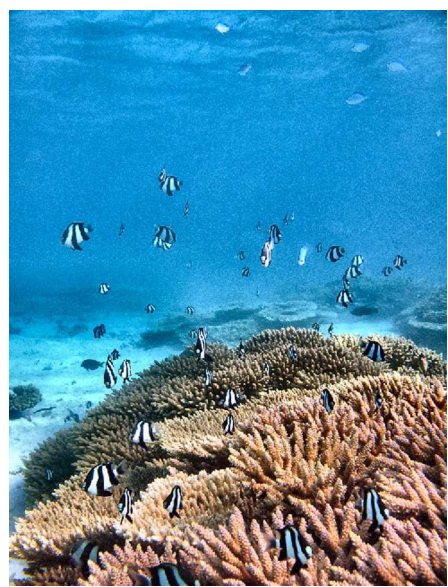

(h) adapthisteq()

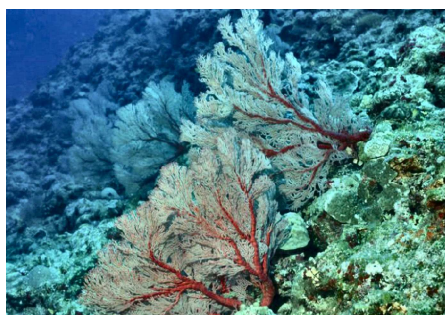

(1) adapthisteq()

Fig. 7. Comparison with histogram equalization.

[5] J. Kopf, B. Neubert, B. Chen, M. Cohen, D. CohenOr, O. Deussen, M. Uyttendaele, and D. Lischinski, "Deep photo: model-based photograph enhancement and viewing," ACM Trans. Graph., vol. 27, no. 5, pp. 1-10, 2008.

[6] S. G. Narasimhan and S. K. Nayar, "Chromatic framework for vision in bad weather," Computer Vision and Pattern Recognition, IEEE Computer Society Conference on, vol. 1, p. 1598, 2000.

[7] R. T. Tan, "Visibility in bad weather from a single image," Computer Vision and Pattern Recognition, IEEE Computer Society Conference on, vol. 0, pp. 1-8, 2008.

[8] R. Fattal, "Single image dehazing," in SIGGRAPH '08: ACM SIGGRAPH 2008 papers. New York, NY, USA: ACM, 2008, pp. 1-9.

[9] K. He, J. Sun, and X. Tang, "Single image haze removal using dark channel prior," Computer Vision and Pattern Recognition, IEEE Computer Society Conference on, vol. 0, pp. 1956-1963, 2009

[10] L. Kratz and K. Nishino, "Factorizing scene albedo and depth from a single foggy image," in ICCV09, 2009, pp. 1701-1708.

[11] S. Q. Duntley, "Light in the sea," J. Opt. Soc. Am., vol. 53, no. 2, pp. 214-233, 1963.

[12] A. Levin, D. Lischinski, and Y. Weiss, "A closed form solution to natural image matting," in CVPR '06: Proceedings of the 2006 IEEE Computer Society Conference on Computer Vision and Pattern Recognition. Washington, DC, USA: IEEE Computer Society, 2006, pp. 61-68.

[13] J. Marroquin, S. Mitter, and T. Poggio, "Probabilistic solution of ill-posed problems in computational vision," Journal of the American Statistical Association, vol. 82, no. 397, pp. 76-89, 1987. [Online]. Available: http://www.jstor.org/stable/2289127

[14] Y. Boykov, O. Veksler, and R. Zabih, "Fast approximate energy minimization via graph cuts," IEEE Trans. Pattern 


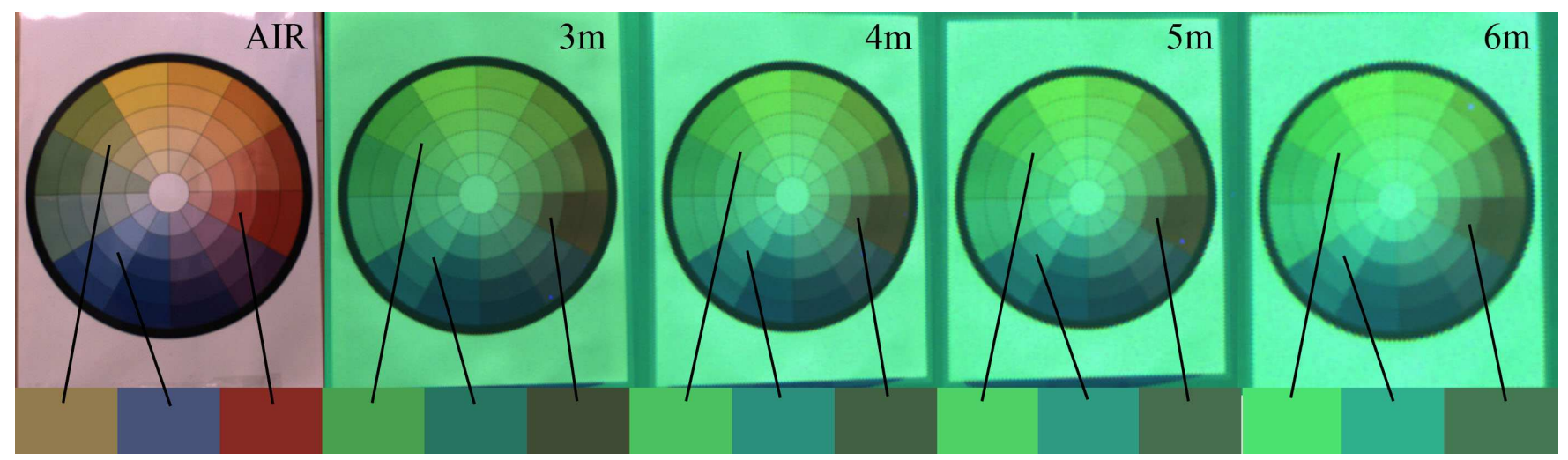

(a) Original images.
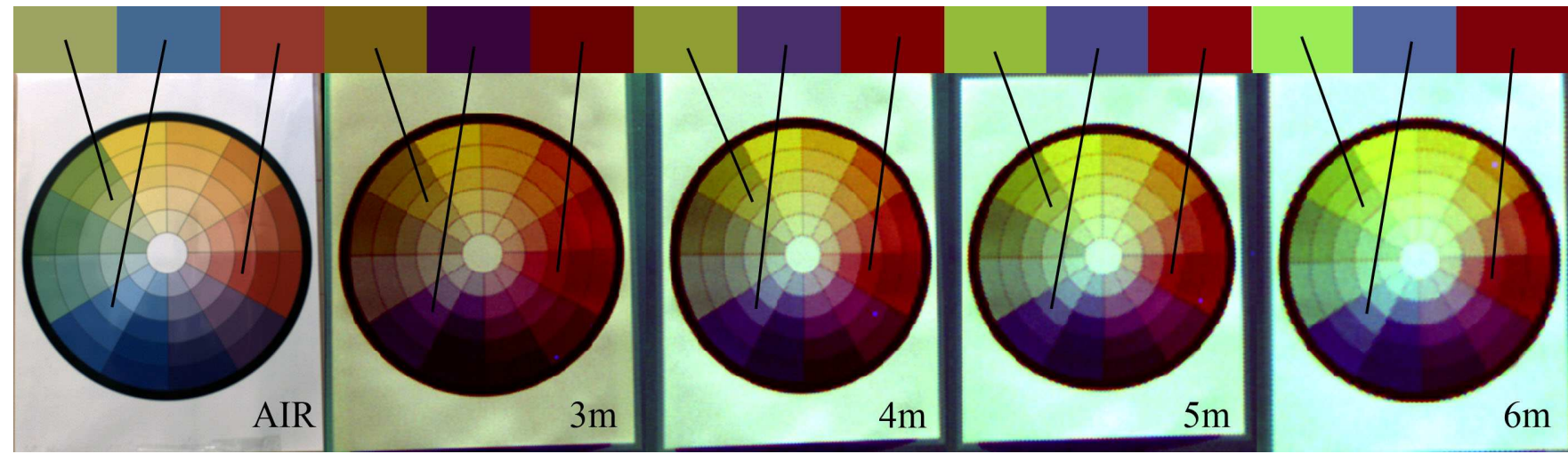

(b) Dehazed images.

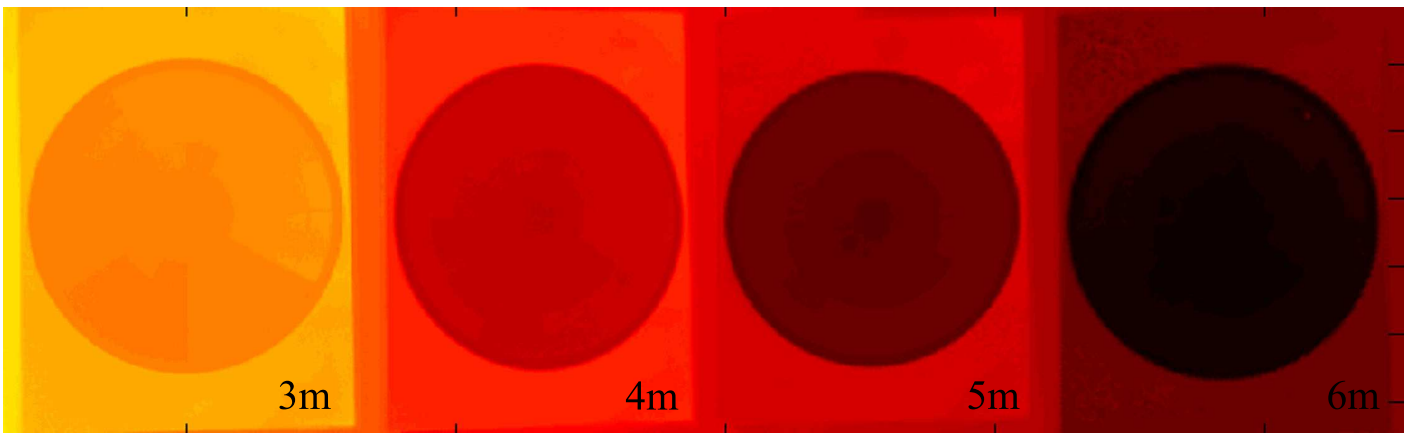

(c) Depth maps.

Fig. 8. Color accuracy test. A printed color wheel was photographed in a test tank at distances 3 to 6 meters from the camera. Three segments of each image of the color wheel have been sampled and displayed for color comparison. The leftmost color wheel image was taken in air as the ground truth. In (a) the image in air is completely unmodified, in (b) the image in air has been white balanced to produce more accurate colors for comparison. Note that in this experiment the airlight was selected manually as the images do not contain a completely haze-obscured region for the airlight estimate as discussed in Section IV.

Anal. Mach. Intell., vol. 23, no. 11, pp. 1222-1239, 2001.

[15] V. Kolmogorov and R. Zabih, "What energy functions can be minimized via graph cuts," IEEE Transactions on Pattern Analysis and Machine Intelligence, vol. 26, pp. 65-81, 2004.

[16] Y. Boykov and V. Kolmogorov, "An experimental comparison of min-cut/max-flow algorithms for energy minimization in vision," IEEE Transactions on Pattern Analysis and Machine Intelligence, vol. 26, pp. 359-374, 2001. 